\title{
The formation of nanoscale clusters - nanofilms / quantum dots predicted using a capillary model of nucleation
}

\author{
Samsudi Sakrani*, Lim Qiao Jie, Yussof Wahab \\ Ibnu Sina Institute for Fundamental Science Studies, Universiti Teknologi Malaysia, 81310 UTM Skudai, Johor, Malaysia \\ *To whome correspondence should be addressed. E-mail: samsudi@difz2.fs.utm.my \\ Received 13 November 2005 \\ http://dx.doi.org/10.11113/mjfas.v1n1.12
}

\begin{abstract}
This paper describes the theoretical and simulation studies of both homogenous and heterogeneous nucleation, the phenomena that refers to the formation of stable nuclei prior to the growth of nanoclusters including nanofilms and quantum dots. Essentially, a single cluster may contain few thousand of atoms, and interaction with the surface may be preceded via processes, such as diffusion, hopping, sorption and coalescences. These complicated physical-chemical phenomena require in-depth theoretical understanding on how the various interacting quantities can be formulated and then resolved using specific mathematical approximation. In the case of a capillary model for heterogeneous nucleation, the nuclei are assumed to be in spherical shapes, which increase in both energies and diameters, and finally reach their critical points and settled to oval shapes prior to dome-like wetting on the substrate, essentially just like water droplet resting on a surface. The net change of energy, $\Delta G$ for the formation of cluster is found to be the functions of nucleus volume, surface area of atomic-nucleus interface, surface area of nucleus-surface interface and energy lost at substrate-atomic interface. The results for $\Delta G, \Delta G^{*}, r^{*}$ and $\Omega$ and their respective changes with $r, s$ and $T$ were obtained and experimentally verified using existing data.
\end{abstract}

| Cluster | Nanofilm | Homogeneous and heterogeneous nucleation | Nucleation rates |

\section{Introduction}

Nucleation is a process that plays a key role in many fields ranging from atmospheric applications to materials science, and its study is currently being stimulated by the development of new experimental and theoretical techniques. It refers to the thermodynamic and kinetic processes that initiate first-order phase transitions in non-equilibrium systems and it also is a process with which the formation of new phases begins and is thus a widely spread phenomenon in both nature and technology [1]. As example, nucleation plays a prominent role in the condensation and evaporation, crystal growth, deposition of thin films and overall crystallization processes [2]. They need to nucleate before a new phase can be formed.

There are many experimental observations reflecting the fact that there are kinetic barriers to first order phase transitions (nucleation process) with metastable phases persisting over large periods of time. The critical nucleus is that fluctuation which lies at the barrier between shrinking and growing regions of the new phase. So the small fluctuations of the new stable phase tend to disappear while large fluctuations tend to grow. The nucleation rate (i.e. the rate of appearance of critical nuclei) then determines the time it takes for a phase 
transition to occur, as the growth beyond the critical nucleus is generally fast compared to its formation rate. As an activated process its rate depends exponentially on the height of the barrier represented by the work of formation of the critical nucleus and the calculation of this value is central in theories of nucleation [3]. Through most of this century, the study of nucleation has been dominated by the classical capillarity approximation.

The process is said to be homogeneous when it takes place in a completely homogeneous phase with no foreign bodies present in the embryo formation. See Fig. 1(a). Because nucleation is generally more rapid if a foreign particulate is present and called heterogeneous nucleation, the latter processes are of most importance in commonly occurring phase transitions - nanofilms/quantum dots. So, it can be said that when any of the latter are present and act to assist the system to get over the activation barrier which we shall see, is imposed by surfacefree-energy considerations, the process is properly called heterogeneous nucleation [4]. Fig. 1 (b).

The principal goal of this paper is to improve our understanding for the capillary model of nucleation theory and also describe the simulation study of both homogenous and heterogeneous nucleation in order to find out clues for optimum parameters required during the early stage of thin film formation.

a)
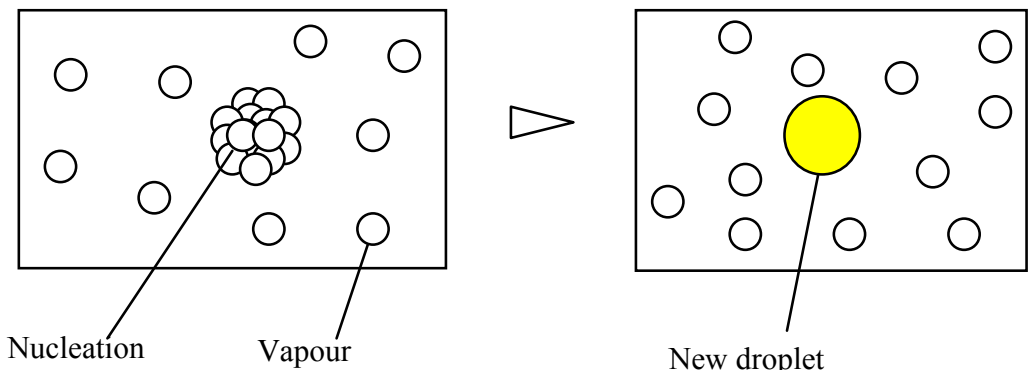

New droplet cluster molecules

b)
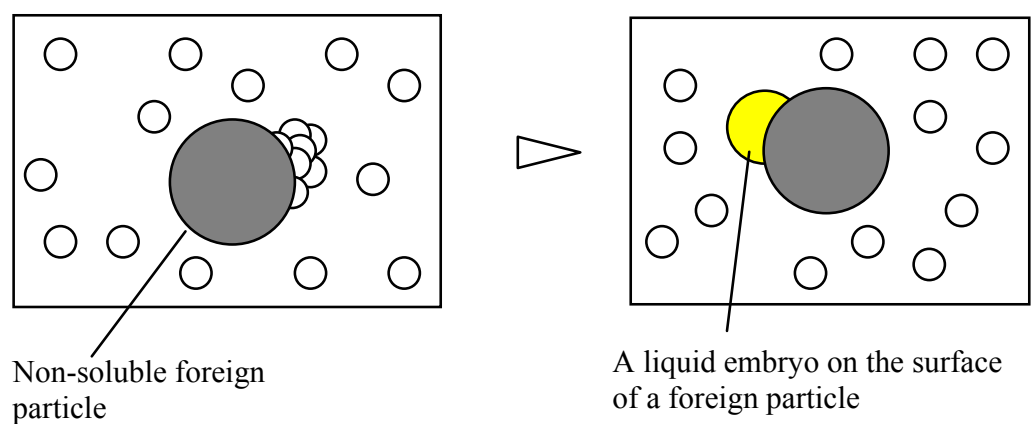

A liquid embryo on the surface of a foreign particle

Fig. 1 (a) Homogeneous nucleation, (b) heterogeneous nucleation.

\section{Methodology of Calculation}

Origins of the theory of nucleation can be traced to the works of Kelvin, who introduced the concept of a critical nucleus and of Gibbs, who calculated the work to form a nucleus. In the 1870s Gibbs showed that the reversible work $\Delta G$ required to form a nucleus of the new phase consists of two terms: A bulk or volumetric term that stabilizes the fragmentary new phase and a surface term that destabilizes it [5]. For homogeneous nucleation, the net change of energy, $\Delta G$ is given by 


$$
\Delta G=4 \pi r^{2} \gamma-\frac{4}{3} \pi r^{3} \Delta G_{v}
$$

where $r$ is the radius of the nucleus, $\gamma$ is surface free energy per unit area and $\Delta G v$ is the free energy change per unit volume, i.e.

$$
\Delta G_{v}=\frac{k T}{v_{0}} \ln \frac{P}{P_{e}}
$$

where $v_{o}$ is molecular volume, $p_{e}$ is saturation vapor pressure of the substance at certain temperature $T, p$ is the vapor pressure and $S=p / p_{e}$ is the supersaturation ratio. The critical radius, $r^{*}$ is the maximum in $\Delta G$; the barrier height or critical energy $\Delta G^{*}$ is equal to

$$
\begin{aligned}
& r^{*}=-\frac{2 \gamma}{\Delta G_{v}}=-\frac{2 w_{o}}{k T \ln S} \\
& \Delta G^{*}=\frac{16 \pi \gamma^{3}}{3\left(\Delta G_{v}\right)^{2}}=\frac{16 \pi \gamma^{3} v_{o}^{2}}{3(k T \ln S)^{2}}
\end{aligned}
$$

Heterogeneous nucleation occurs at impurities interface. A simple theory can be established from the concept of surface tension occurring in liquid when its surface is in contact with a capillary tube, also known as capillary model of nucleation. Fig. 2 shows the mathematical configuration of a dome-like shape.

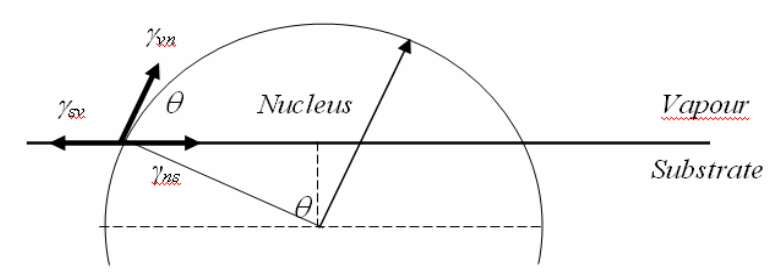

Fig. 2 A dome-like shape nucleus.

So that the total energy changes is

$$
\Delta G=\frac{\pi\left(2-3 \cos \theta+\cos ^{3} \theta\right)}{3} r^{3} \Delta G_{v}+2 \pi(1-\cos \theta) \gamma_{v n} r^{2}+\pi \sin ^{2} \theta\left(\gamma_{n s}-\gamma_{s v}\right) r^{2}
$$

where $\gamma_{v n}$ is the surface energy per unit area between vapour-nucleus, $\gamma_{s v}$ is the surface energy per unit area between substrate-vapour, $\gamma_{n s}$ is the surface energy per unit area between nucleus-substrate, $\theta$ is the contact angle and $r$ is the diameter of spherical nucleus. By taking differentiation of $\mathrm{d}(\Delta G) / \mathrm{d} r=0$, the critical radius $r^{*}$ and critical energy $\Delta G^{*}$ are

$$
r^{*}=\frac{-2\left(a_{1} \gamma_{f v}+a_{2} \gamma_{f s}-a_{2} \gamma_{s v}\right)}{3 a_{3} \Delta G_{v}}
$$




$$
\Delta G^{*}=\frac{4\left(a_{1} \gamma_{f v}+a_{2} \gamma_{f s}-a_{2} \gamma_{s v}\right)^{3}}{27 a_{3}^{2} \Delta G_{v}^{2}}
$$

where

$$
a_{1}=2 \pi(1-\cos \theta) \quad, \quad a_{2}=\pi \sin ^{2} \theta \quad \text { and } \quad a_{3}=\frac{\pi\left(2-3 \cos \theta+\cos ^{3} \theta\right)}{3}
$$

The nucleation rate, $J$ is defined as the frequency of appearance at time $t$ of nuclei per unit volume or area of the system under consideration. For homogeneous and heterogeneous nucleation, nucleation rate is the combination between population of critical nuclei, critical surface area of nuclei (Fig. 3) and flux of atom impingement that equal to:

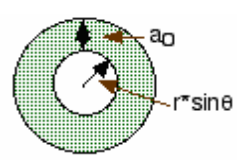

Fig. 3 Surface area of nuclei

$$
J_{\text {homo }}=\left[n_{s} \exp \left(-\frac{\Delta G *}{k T}\right)\right] 4 \pi(r *)^{2}\left[\frac{\alpha\left(\frac{P}{P_{e}}-1\right) N_{A}}{\sqrt{2 \pi M R T}}\right]
$$

$$
J_{\text {hetero }}=\left[\sqrt{2 \pi} r^{*} a_{o} \sin \theta\right] \frac{P N_{A}}{\sqrt{M R T}}\left[n_{s} \exp \left(\frac{E_{\text {des }}-E_{\text {diff }}-\Delta G^{*}}{2 k T}\right)\right]
$$

where $n_{s}$ is the density of all nuclei, $\alpha$ is the sticking coefficient, $N_{A}$ is the Nombor Avagadro, $M$ is the atomic weight and $R$ is gas constant, $P$ is the vapor pressure, $E_{\text {des }}$ is the desorption energy and $E_{\text {diff }}$ is the diffusion energy.

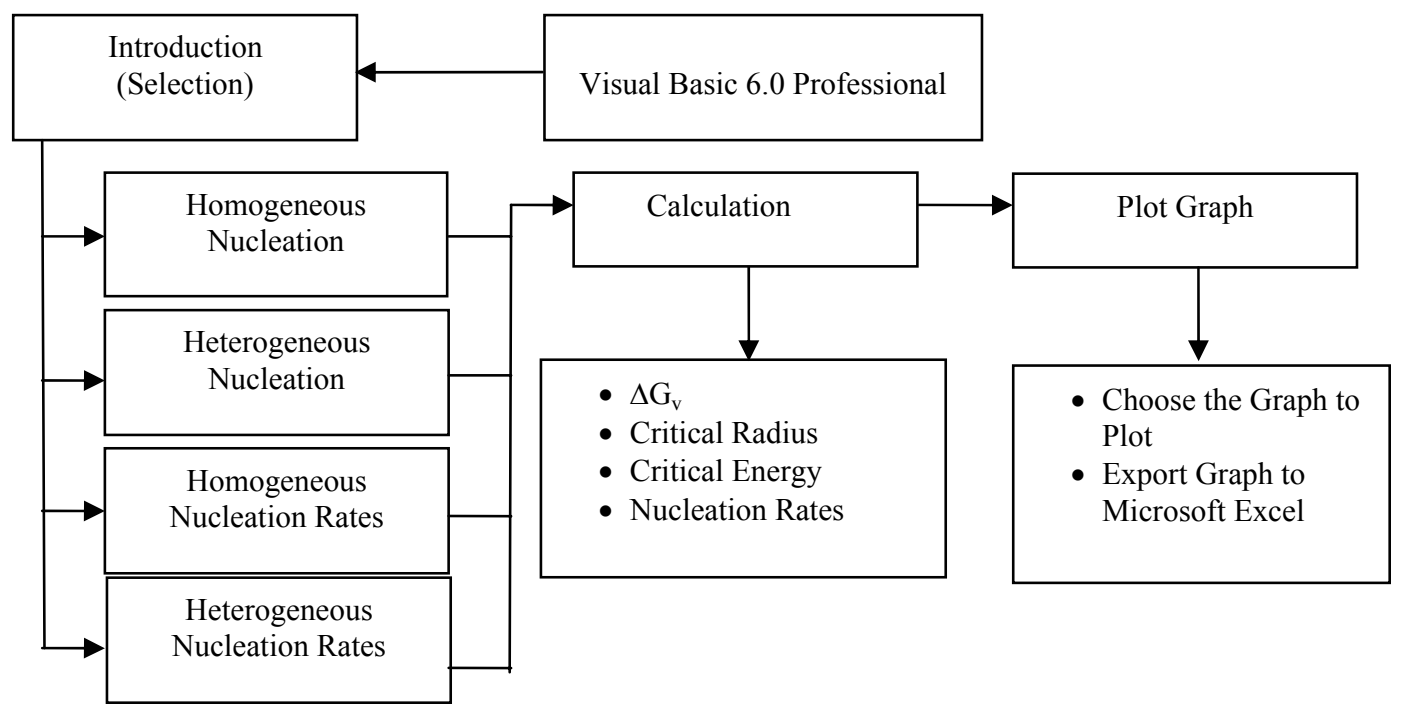

Fig. 4 Flow chart showing the simulation process 
Generally, the rate of nucleation, $J$, in Classical Nucleation Theory is an exponential function of $\Delta G^{*}$, i.e.,

$$
J=J_{o} \exp \left(\frac{-\Delta G^{*}}{R T}\right)
$$

where the pre-exponential factor $J_{0}$ is not very dependent upon the supersaturation, compared with the exponential factor, and therefore one can consider $J_{o}$ as nearly a constant. Typical values of $J_{o}$ for homogeneous nucleation are $10^{25}-10^{35} \mathrm{~cm}^{-3} \mathrm{~s}^{-1}$. $J_{o}$ for heterogeneous nucleation is depends on the nucleation process. As example, $J_{o}$ for the condensation from vapour nucleation process is $10^{17} \mathrm{~cm}^{-2} \mathrm{~s}^{-1}$.

By knowing the concept and simple equation of nucleation theory, a simulation program was conducted using Microsoft Visual Basic 6.0 Professional, Microsoft Windows XP Home Edition and Microsoft Excel 2003. The flow chart of simulation program is shown in Fig. 4.

\section{Results and Discussion}

The classical theory of nucleation is a reliable basis for the quantitative description of nucleation phenomena up to now. Based on the equation above, the simulation of nucleation theory would require the knowledge of additional material parameters. But in this paper, the results of simulation is based on the assumption value of material parameters and not specific to any material.
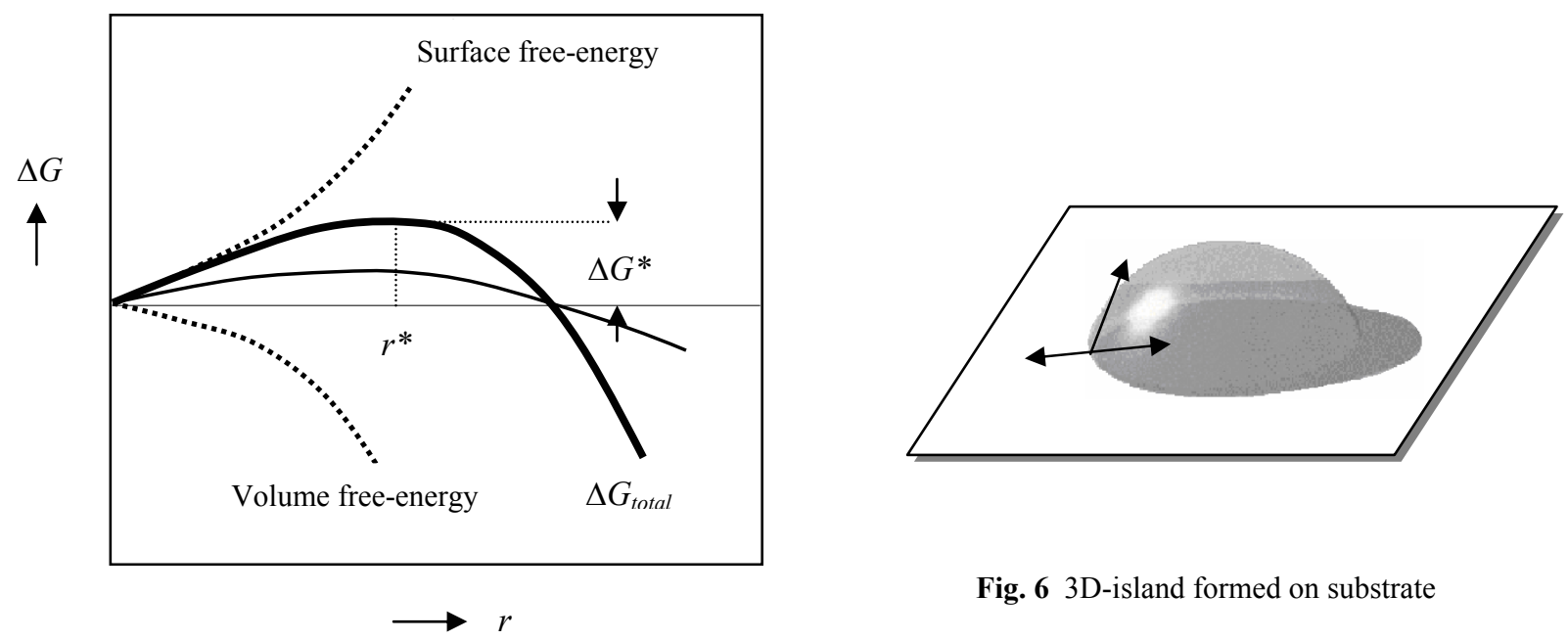

Fig. 6 3D-island formed on substrate

Fig. 5 Graph of $\Delta G$ versus $r$ (Homogeneous Nucleation)

Figure 5 shows the Gibbs free energy change as function of droplet radius for homogeneous nucleation. The total free energy change is the combination of surface free-energy change which goes up as the square of radius and the volume free-energy change which goes up as the cube of radius. From Figure 5, $\Delta G$ displays a maximum at some critical size $r^{*}$ given by equation (1). An embryo of radius $r^{*}$ maybe in metastable equilibrium with surrounding vapor, if some more atoms join the critical nucleus its radius increases and in turn its equilibrium vapor pressure becomes smaller than the one available in the system. Then the probability of decay becomes smaller than the probability of growth and the nucleus should grow further after critical radius [6]. Figure 5 also shows a maximum value of $\Delta G$ which gives the height of the energy barrier which should be overcome for 
condensation or growth process to take place. Figure 6 shows a 3-dimentional view of the dome-like shape of the cluster, balanced by three forces acting on it.

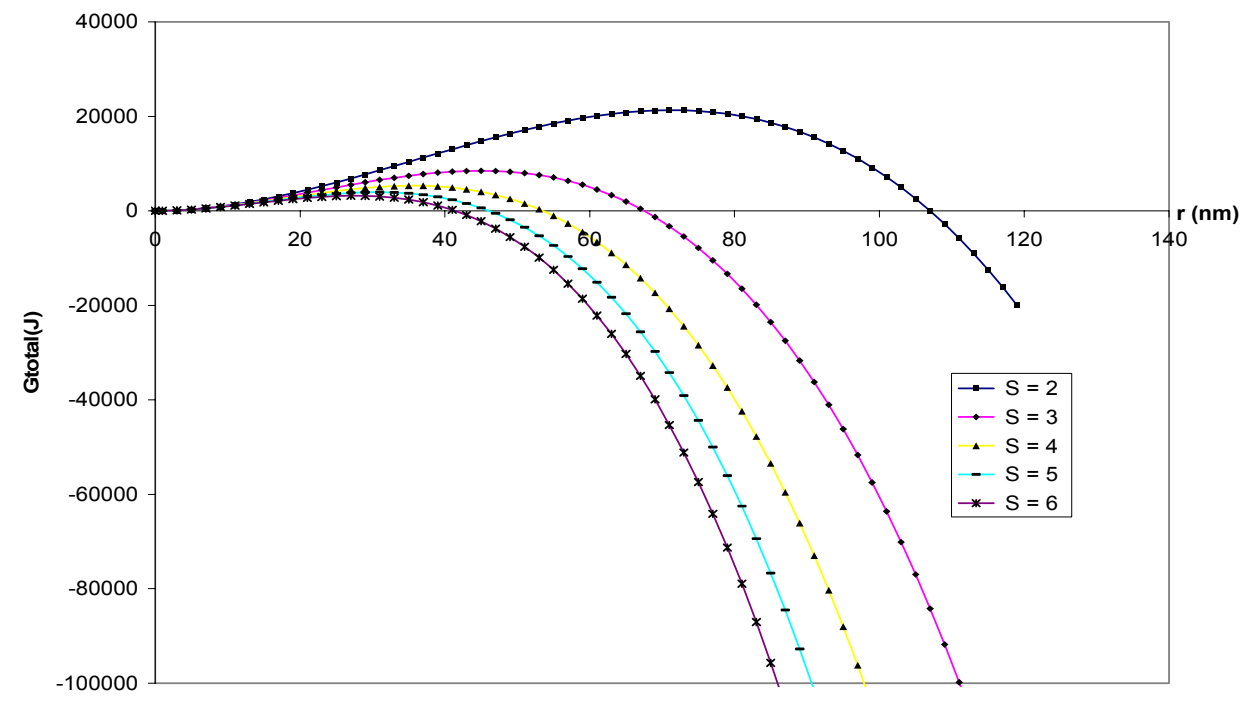

Fig. 7 Graph of $\Delta G$ versus $r$ at different supersaturation ratios, $S$ for homogeneous nucleation

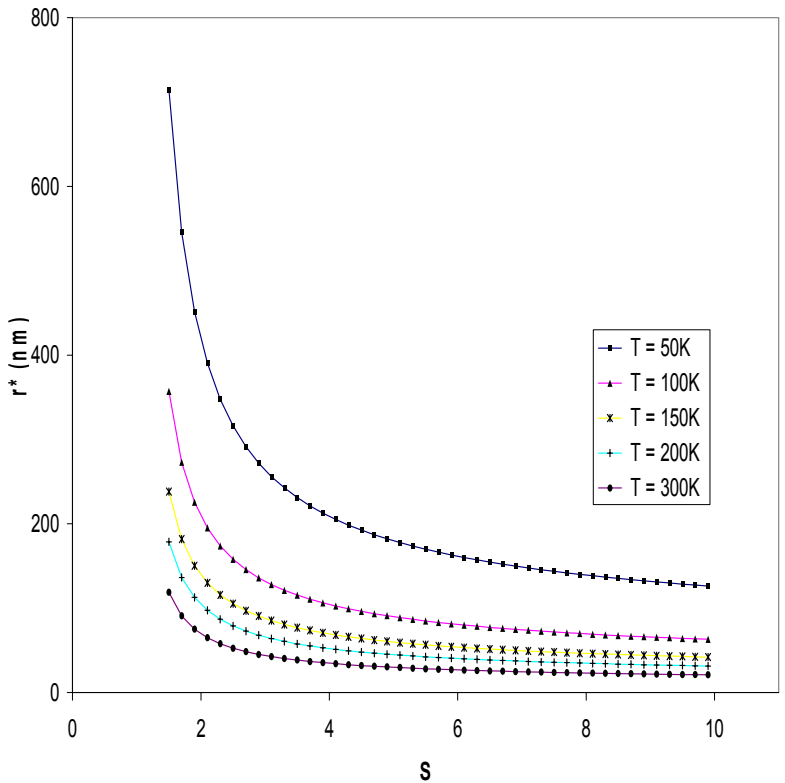

Fig. 8 Graph of critical radius, $r *$ versus superaturation ratio, $S$ at different temperatures for homogeneous nucleation

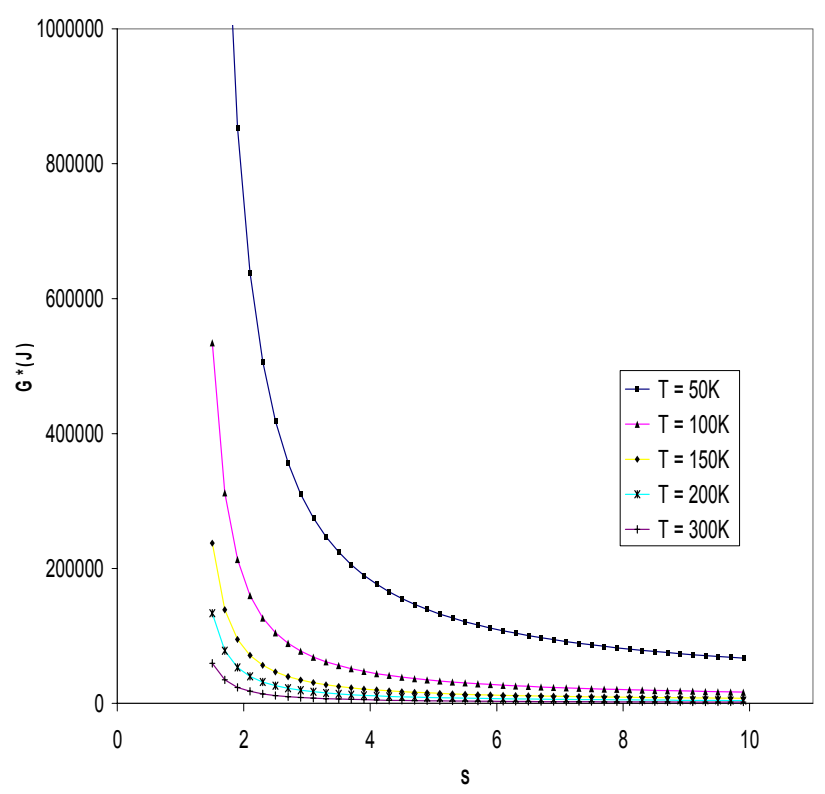

Fig. 9 Graph of critical energy, $\Delta G^{*}$ versus superaturation ratio, $S$ at different temperatures for homogeneous nucleation 
The supersaturation ratio, $S$ is an important parameter which influence the free energy change $\Delta G$, critical radius $r^{*}$ and critical energy $\Delta \mathrm{G}^{*}$ for a fixed $T$ as described by equations (2), (3) and (4), the. Figure 7 shows the free energy change as function of radius at different $S$ for fixed $T$. The relation reveals the very important rule that the larger the supersaturation ratio, $\mathrm{S}$, the smaller the critical radius $r^{*}$ and critical energy $\Delta G^{*}$. As supersaturation ratio, $S$, increase, it will raise the molecular bombardment rate and reduce $r^{*}$ and the attendant height of the activation energy barrier $\Delta G^{*}$ to such extent that the probability of some sub-critical embryo fortuitously growing to supercritical size in a short time approaches unity [6]. Homogeneous nucleation becomes an effective process at a sufficient $S$ value and phase transition can finally begin. To make it clearly, Figures 8 and 9 show the critical radius $r^{*}$ and critical energy $\Delta G^{*}$ as a function of Supersaturation ratio varying with temperature deduced from equations (3) and (4). It is evidence that $r^{*}$ and $\Delta G^{*}$ will decrease when $S$ is increased. The higher the temperature condition, $T$, the smaller the critical radius $r^{*}$ and critical energy $\Delta G^{*}$ at each supersaturation ratio, $S$.

In heterogeneous nucleation the nuclei can be formed at preferential sites (e.g. substrate, mould wall, impurities or catalysts, etc.). The graph of free energy change versus radius was plotted using equation (5) as shown in Fig. 10. The result indicates that $\Delta G$ for heterogeneous nucleation displays maximum value of critical energy, $\Delta G^{*}$ and critical radius, $r^{*}$. To make the theory of heterogeneous nucleation applicable, we assumed that substrate is inert or non-reacting and the following conditions are obeyed, i.e., $\gamma_{s v} \approx 0$ and $\gamma_{v n} \approx \gamma_{n s}$. Then the critical nucleus size, $r^{*}$ is the same for both heterogeneous and homogeneous nucleation, but the $\Delta G^{*}$ for heterogeneous nucleation is equal to:

$$
\Delta G^{*}=\frac{16 \pi \gamma_{v n}^{3}}{3\left(\Delta G_{v}\right)^{3}} S(\theta) \quad \text { where } \quad S(\theta)=\left(\frac{2-3 \cos \theta+\cos ^{3} \theta}{4}\right)
$$

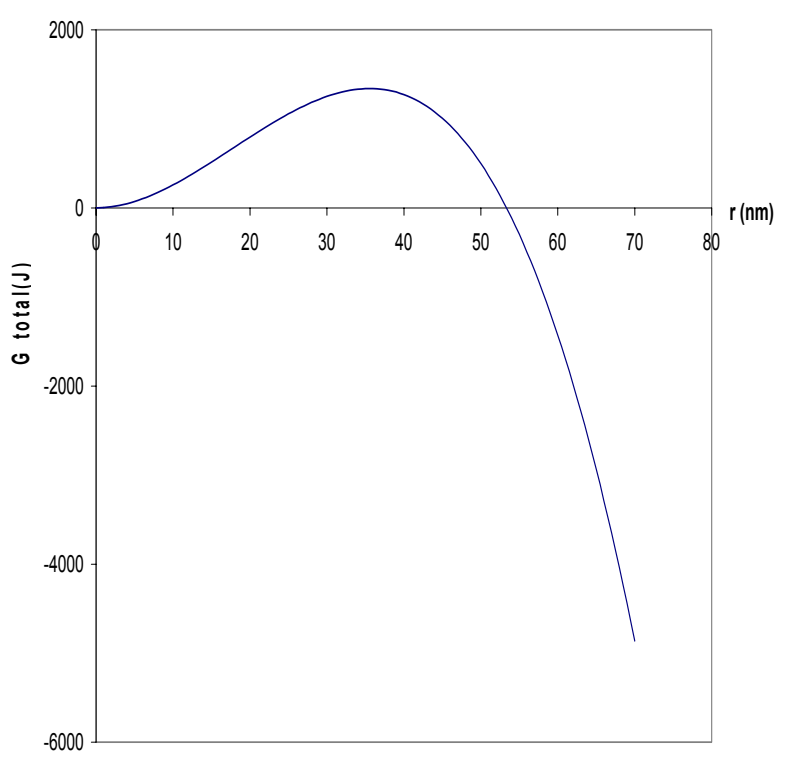

Fig. 10 Graph of free energy, $\Delta G$ versus radius, $r$ for heterogeneous nucleation

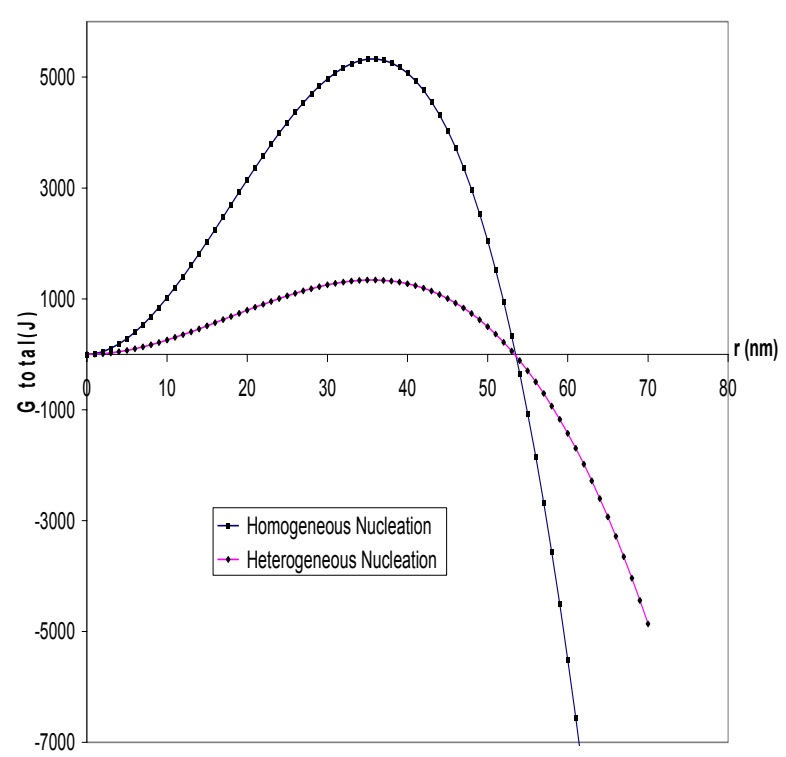

Fig. 11 Graph of free energy, $\Delta G$ versus radius, $r$ between homogeneous and heterogeneous nucleation 
Figure 11 shows the free energy change as function of radius between homogeneous and heterogeneous nucleation. It is evidence that the critical radius $r^{*}$ is the same for both homogeneous and heterogeneous nucleation. However, due to the shape factor $S(\theta)$, the value of critical energy, $\Delta G^{*}$ can be significantly smaller for heterogeneous nucleation, depending on the wetting angle, $\theta$. So, Heterogeneous nucleation is a nucleation event which can occur more easily than the homogeneous nucleation due to the extra surface energy terms that changes the free energy for nuclei formation. The extra surface energy term comes from extra surfaces generated by things such as dirt particles or the inner wall of the mold block during casting process. In a way, these factors which contribute extra surfaces can be view as acting as a "catalyst" for nucleation [7].

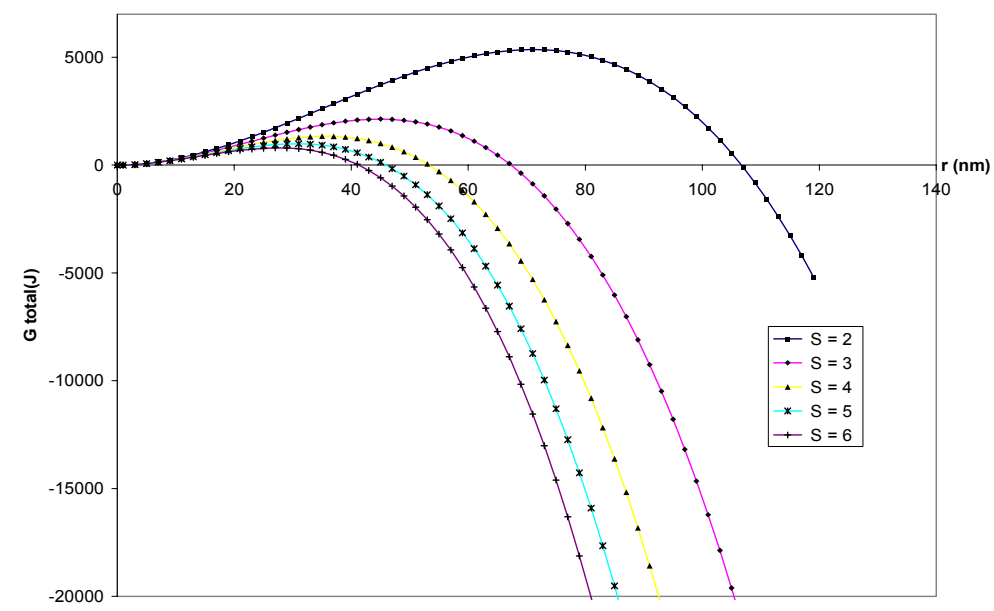

Fig. 12 Graph of free energy, $\Delta G$ versus radius, $r$ at different superaturation ratios, $S$ for heterogeneous nucleation

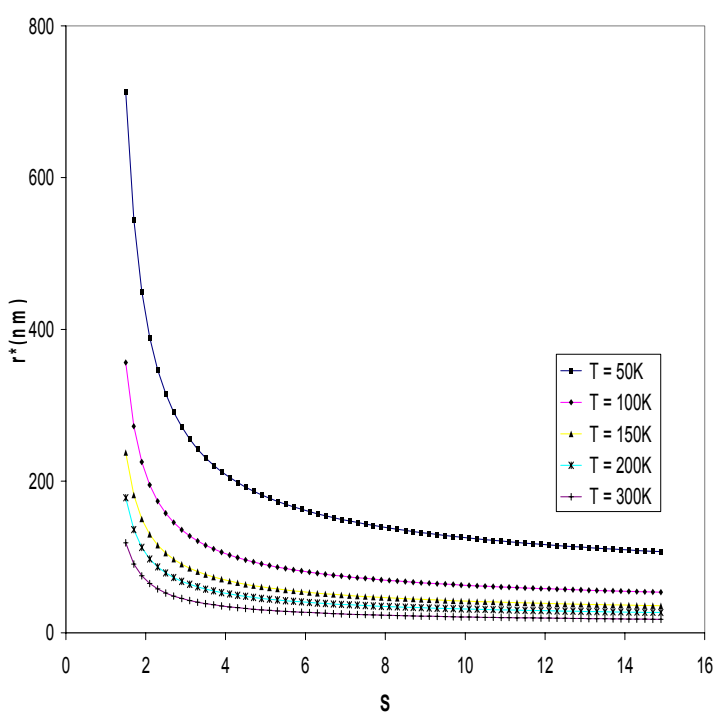

Fig. 13 Graph Critical Radius $(r *)$ versus superaturation ratio, $S$ with different temperature for heterogeneous nucleation

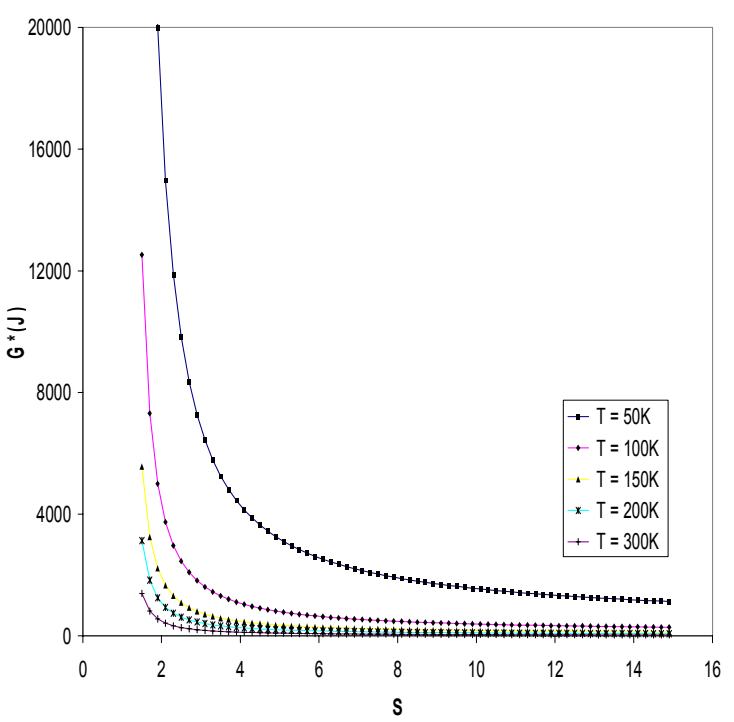

Fig. 14 Graph of critical energy, $\Delta G^{*}$ versus superaturation ratio, $S$ with different temperature for heterogeneous nucleation 
Like the homogeneous nucleation, the values of $r^{*}$ and $\Delta \mathrm{G}^{*}$ in heterogeneous nucleation are dependence on the supersaturation ratio for a fixed temperature. Figure 12 shows the plot for free energy change versus radius by varying the supersaturation ratio, $S$. We found that the larger the supersaturation ratio, $S$, the smaller the critical radius $r^{*}$ and critical energy $\Delta G^{*}$. From Figures 13 and 14, we can observe that $r^{*}$ and $\Delta G^{*}$ decrease with an increase of temperature at a certain supersaturation ratio. Thus, the effect of temperature also is very significant in influencing the nucleation process.

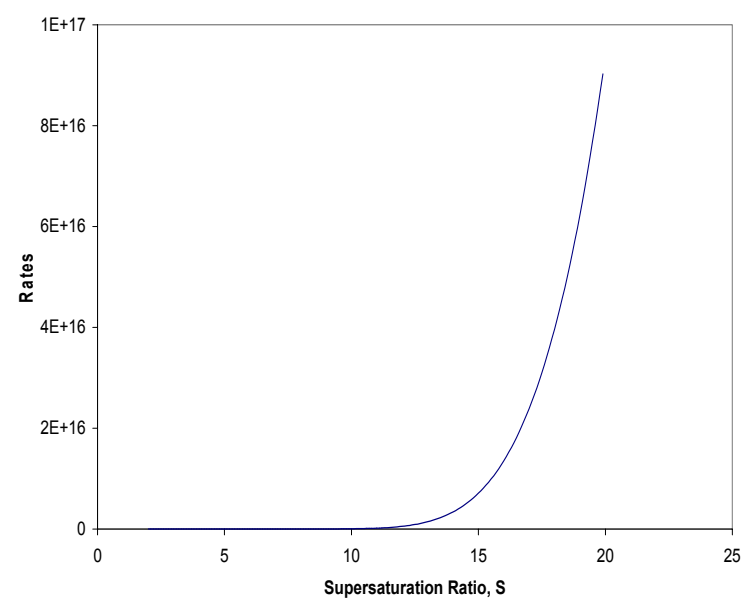

Fig. 15 Graph of nucleation rates, $J$ versus superaturation ratio, $S$ for homogeneous nucleation

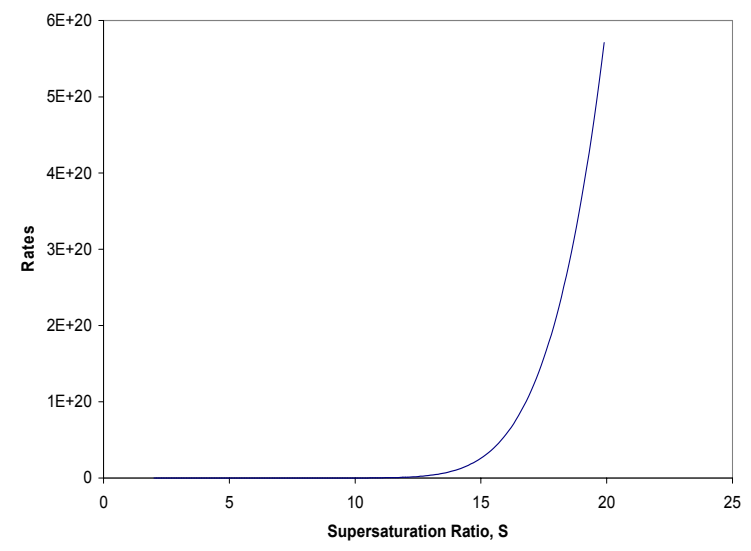

Fig. 17 Graph of nucleation rates, $J$ versus superaturation ratio, $S$ for heterogeneous nucleation

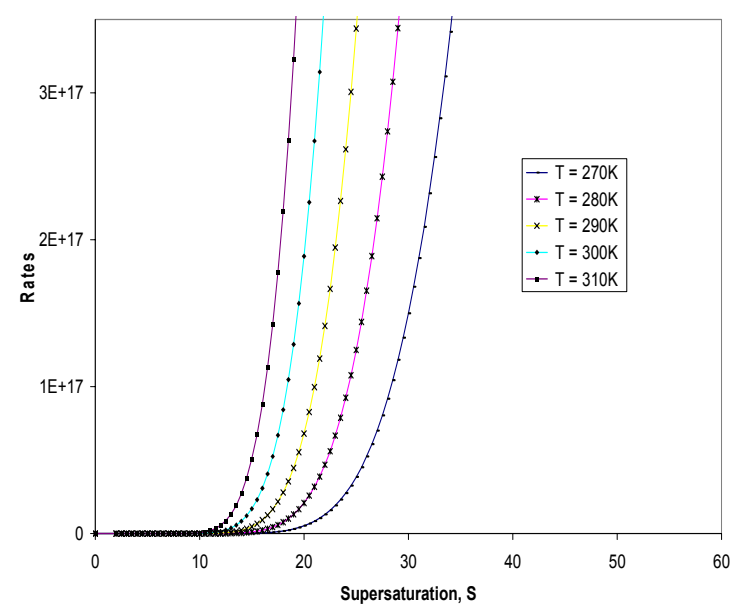

Fig. 16 Graph of nucleation rates, $J$ versus superaturation ratio, $S$ with different temperature for homogeneous nucleation

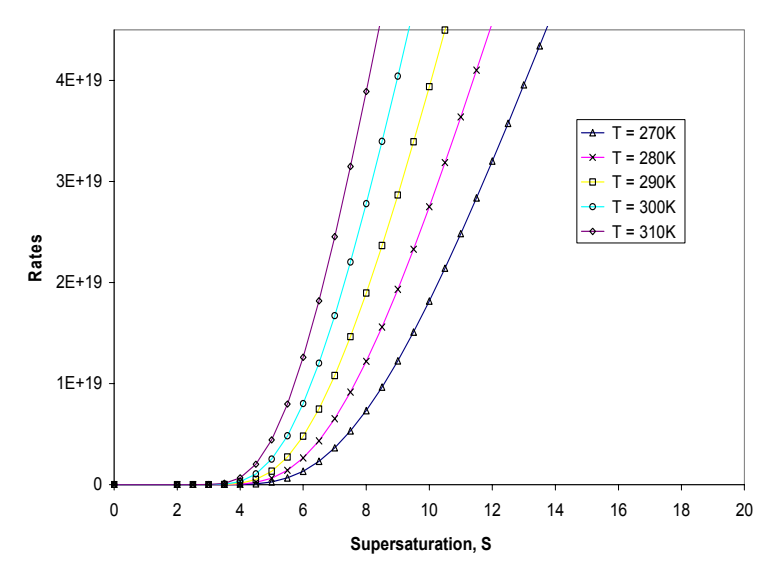

Fig. 18 Graph of nucleation rates, $J$ versus superaturation ratio, $S$ with different temperature for heterogeneous nucleation 
Nucleation is a random process; the number of nuclei formed in a fixed interval of time is a random quantity and is subject to statistical laws [8]. However, the average values can be calculated and are subject to the kinetic theory of nucleation. Figure 15 shows the nucleation rates as a function of supersaturation ratio and the effects of varying temperatures is shown in Figure 16. These graphs were obtained for homogenous nucleation based on equation (8). All constant parameters, such as density of nuclei, sticking coefficient, atomic weight and others have been assumed for the purpose of plotting the graph. In case of heterogeneous nucleation as described by equation (9), the graphs (Figs. 17 and 18) exhibit similar trends with higher rates of the order $10^{19}$.

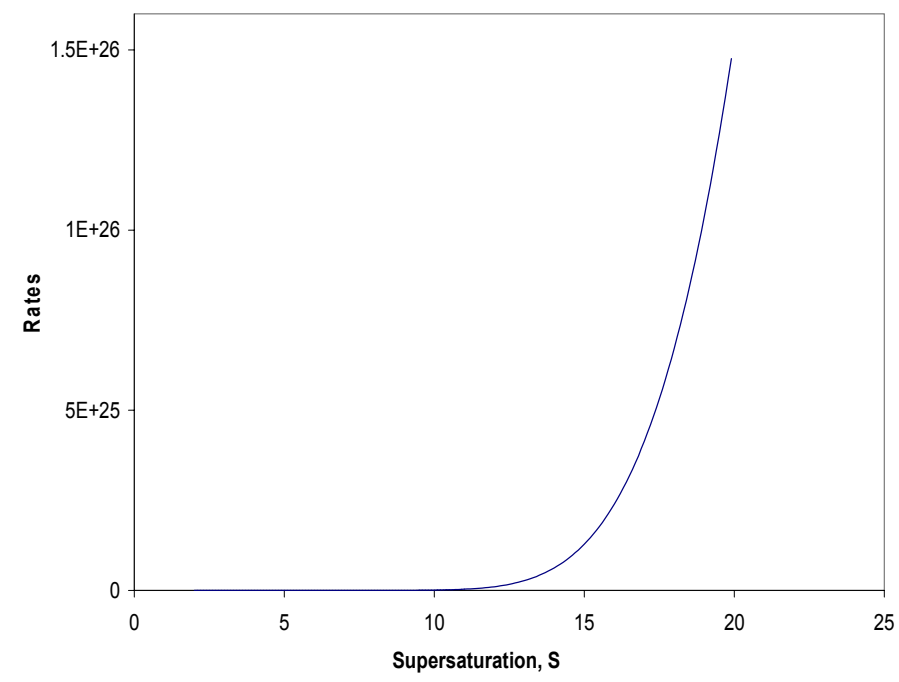

Fig. 19 Graph of nucleation rates, $J$ versus superaturation ratio, $S$ plotted using equation (10)

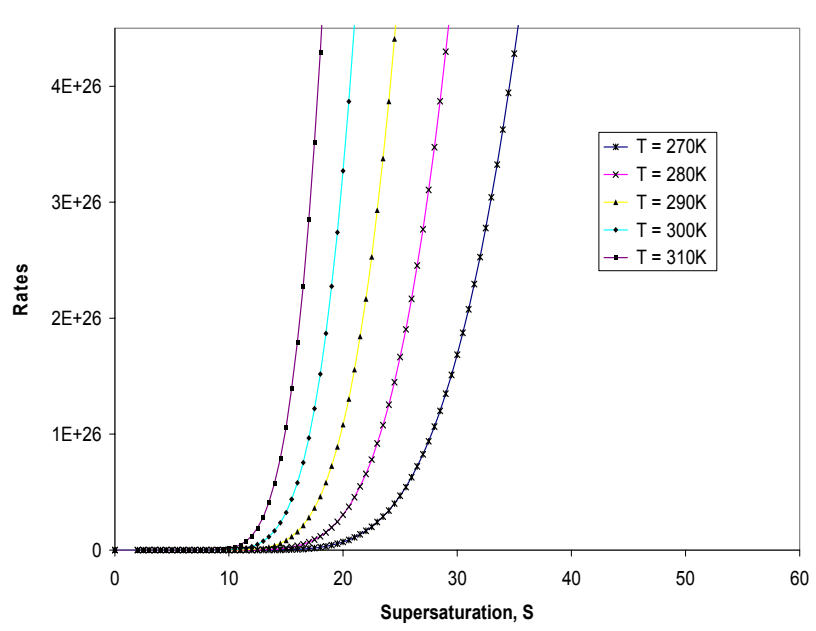

Fig. 21 Graph of nucleation rates, $J$ versus superaturation ratio, $S$ at different temperatures plotted using equation (10)

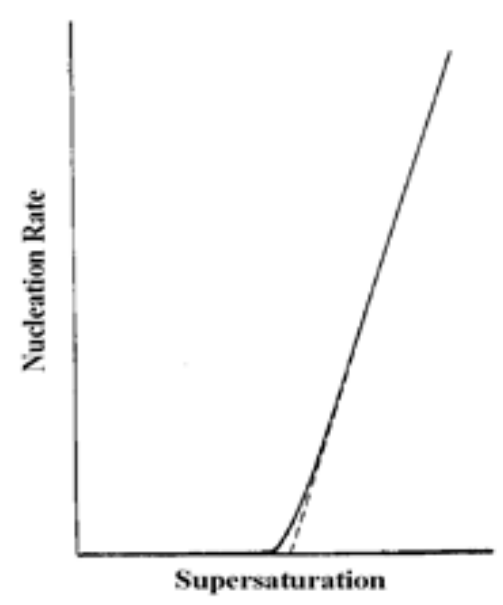

Fig. 20 Theoretical graph [10] of nucleation rates, $J$ versus superaturation ratio, $S$

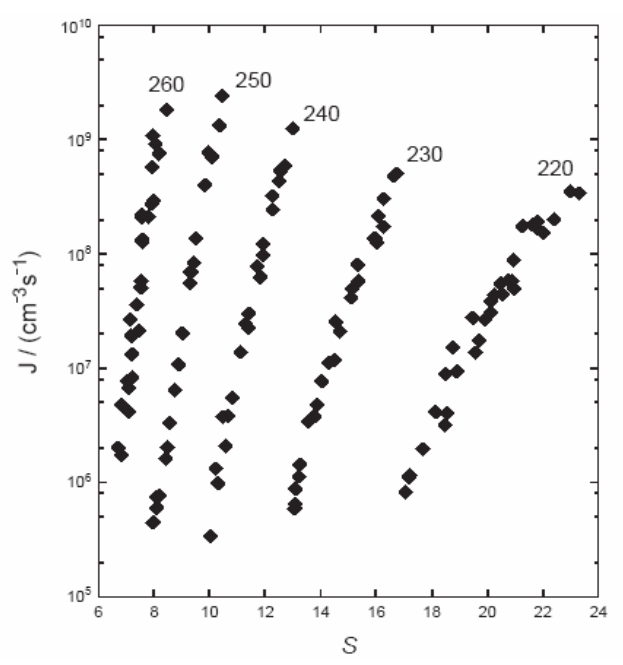

Fig. 22 The measured nucleation rates $J$ for the condensation of water as a function of supersaturation $S$ at different temperatures 
Referring to the equation (10), it is a general expression valid for all possible cases of nucleation and can be used in any particular case. Based on the equation (10) and the assumption that pre-exponential factor value is $10^{25} \mathrm{~cm}^{-3} \mathrm{~s}^{-1}$, the graph of nucleation rates versus supersaturation ratio was obtained and shown in Figure 19 . It appears that nucleation rates remains negligibly small until the supersaturation reaches a critical value, the Ostwald metastable limit, at which point nucleation rates suddenly and dramatically increases. Hence, this critical supersaturation level can be arbitrarily set as the rate at which the rate is 1 nucleus $\mathrm{cm}^{-3} \mathrm{sec}^{-1}$ (or any other suitable experimentally detectable limit) with little loss of accuracy [9]. The plot is in a good agreement with the predicted graph (Fig. 20) reported elsewhere [10].

Figure 21 shows the nucleation rates as function of supersaturation ratio at different temperature which was plotted based to the equation (10). Nucleation rate increases very steeply as a function of the Supersaturation at any given temperature. But when the temperature is higher, the critical value of supersaturation becomes smaller. So, the simulation results of nucleation rates are in good agreement with Wolk and Strey who reported the experimental result for the nucleation rates, $J$ for the condensation of water as a function of supersaturation $\mathrm{S}$ at different temperatures [11] as shown in Figure 22.

\section{Conclusion}

In conclusion, the graphs of simulation results are in a good agreement with the theory and partly verified by the experimental evidence. The Gibbs free energy change, $\Delta G$ revealed a maximum point, $\Delta G^{*}$ at a critical nucleus size, $\mathrm{r}^{*}$ for both homogeneous and heterogeneous nucleation. The effects of the supersaturation ratio and temperature parameters are significant for quantitative descriptions of homogeneous and heterogeneous nucleation. There was a critical supersaturation value for nucleation rates to increase suddenly and dramatically; this was normally referred to the rate at which the rate is 1 nucleus $\mathrm{cm}^{-3} \mathrm{sec}^{-1}$. This simulation program can be used to predict the optimum parameters required during the early stage of thin film nucleation when the appropriate parameters are given.

\section{Acknowledgements}

The authors wish to express their thanks to the Universiti Teknologi Malaysia and Government of Malaysia for financing this project through Intensification of Research in Priority Area (IRPA) of Vot 74542.

\section{References}

[1] A. Obeidat, J. S. Li, and G. Wilemski, J. Chem. Phys., 121 (2004) 9510.

[2] D. Kashchiev, Nucleation: Basic Theory with Applications, Oxford: Butterworth-Heinemann, 2000.

[3] D. W. Oxtoby, Acc. Chem. Res., 31 (1998) 91.

[4] J. L. Katz, Pure Appl. Chern., 64 (1992) 1661.

[5] J. W. Gibbs, The Scientific Papers of J. W. Gibbs (Dover, New York) 1.1 (1961) 253.

[6] F. F. Abraham, Homogeneous Nucleation Theory, New York: Academic Press Inc, 1974.

[7] Lewis \& J.C.Anderson, Nucleation and Growth of Thin Films, New York: Academic Press Inc, 1978.

[8] G. Madras, B. J. McCoy, Chemical Engineering Science 59 (2004) 2753.

[9] V. Talanquer, J. Chem. Ed., 79 (2002) 877.

[10] V. Markov, Crystal Growth for Beginners, Singapore: World Scientific, 1995 (Rep 1996).

[11] J. P. Leonard, Nucleation Rate Measurement in the $\mathrm{Si}_{-} \mathrm{SiO}_{2}$ Thin Film System, UMI number: 9970228 , 2000. 